Article (refereed)

Quincey, Paul; Butterfield, David; Green, David; Coyle, Mhairi; Cape, J. Neil. 2009 An evaluation of measurement methods for organic, elemental and black carbon in ambient air monitoring sites. Atmospheric Environment, 43 (32). 5085-5091. 10.1016/i.atmosenv.2009.06.041

Crown copyright @ 2009 Published by Elsevier Ltd

This version available at http://nora.nerc.ac.uk/7602/

NERC has developed NORA to enable users to access research outputs wholly or partially funded by NERC. Copyright and other rights for material on this site are retained by the authors and/or other rights owners. Users should read the terms and conditions of use of this material at http://nora.nerc.ac.uk/policies.html\#access

This document is the author's final manuscript version of the journal article, incorporating any revisions agreed during the peer review process. Some differences between this and the publisher's version remain. You are advised to consult the publisher's version if you wish to cite from this article.

www.elsevier.com 


\title{
An evaluation of measurement methods for organic, elemental and black carbon in ambient air monitoring sites
}

Paul Quincey and David Butterfield

Analytical Science Team, National Physical Laboratory, Hampton Road, Teddington, Middlesex, TW11 0LW, UK.

\section{David Green}

Environmental Research Group, King’s College London, Franklin-Wilkins Building, 150 Stamford Street, London SE1 9NN.

\section{Mhairi Coyle , J. Neil Cape}

Centre for Ecology \& Hydrology, Bush Estate, Penicuik, Midlothian, EH26 0QB, UK

Tel.: +44 (0)20 89436788 Fax : +44 (0)20 89436755

e-mail: paul.quincey@npl.co.uk

\begin{abstract}
The carbonaceous components of Particulate Matter samples form a substantial fraction of their total mass, but their quantification depends strongly on the instruments and methods used. United Kingdom monitoring networks have provided many relevant data sets that are already in the public domain. Specifically, hourly organic carbon (OC) and elemental carbon (EC) were determined at four sites between 2003 and 2007 using Rupprecht and Pattashnik (R \& P) 5400 automatic instruments. Since 2007, daily OC/EC measurements have been made by manual thermo-optical analysis of filter samples using a Sunset Laboratory Carbon Aerosol Analysis instrument. In parallel, long term daily measurements of Black Smoke, a
\end{abstract}


quantity directly linked to black carbon (measured by aethalometers) and indirectly related to elemental carbon, have been made at many sites. The measurement issues associated with these techniques are evaluated in the context of UK measurements, making use of several sets of parallel data, with the aim of aiding the interpretation of network results. From the results available, the main conclusions are that the $\mathrm{R} \& \mathrm{P}$ 5400 instruments greatly underread EC and total carbon $(\mathrm{TC}=\mathrm{OC}+\mathrm{EC})$ at kerbside sites, probably due to the fact that the smaller particles are not sampled by the instrument; the R \& P 5400 instrument is inherently difficult to characterise, so that all quantitative results need to be treated with caution; both aethalometer and Black Smoke (converted to black carbon) measurements can show reasonable agreement with elemental carbon results; and manual thermo-optical OC/EC results may underread EC (and hence overread OC), whether either transmittance or reflectance is used for the pyrolysis correction, and this effect is significant at rural sites.

\section{Keywords}

Black Smoke, Black Carbon, Organic Carbon, Elemental Carbon, air quality, UK

\section{Introduction}

Carbonaceous particles are a major component of airborne particulate matter (PM), forming around $40 \%$ of average roadside $\mathrm{PM}_{10}$ in the UK, according to AQEG (2005) based on the work of Harrison et al (2003).

The analysis of the carbonaceous component of particles has traditionally distinguished between "elemental carbon" (EC) and "organic carbon" (OC), where the EC is loosely associated with the terms "soot" and "black carbon", and OC is 
everything else. No assumptions are made about the compounds present, with both quantities being expressed as the mass of particle-bound carbon in a unit volume of air. In practice the total carbon $(\mathrm{TC}=\mathrm{EC}+\mathrm{OC})$ on filter samples is relatively well defined and can be measured by the thermal volatilisation of carbonaceous material combined with quantitative detection of the carbon produced, for example using a methanator and a flame ionisation detector. There is a separable issue of whether the material collected on filters is representative of carbonaceous material in the ambient atmosphere, as it is well known that semi-volatile organic compounds, for example, can lead to significant variations in collected material depending on the collection conditions (eg Turpin et al, 1993; Eatough et al, 1993). The majority of the methods described here are based on filter samples. The control of artifacts is an active area of study, for example within the ongoing European EUSAAR project. However, the issue of sampling artifacts is not the main subject of this paper and will not be addressed further. Unlike TC, the $\mathrm{OC}$ and EC fractions are operationally defined using semi-empirical methods described in more detail below.

The determination of "black carbon" is based on entirely different physical principles, using optical rather than chemical techniques. Within the United Kingdom, measurements of Black Smoke Index, based on the reflectance of a daily filter sample, have been made since the 1920s. Although expressed in units of $\mu \mathrm{g} / \mathrm{m}^{3}$, the convention used to produce the Black Smoke Index (ISO 9835, 1993) means that the reported results are very much larger than any determination of EC. More recent optical techniques, based on the transmittance of a filter sample, estimate black carbon by using a mass extinction coefficient to give a realistic measure of soot in $\mu \mathrm{g} / \mathrm{m}^{3}$ (eg Hansen et al., 1984). Quincey (2007) has shown that the two filter-based optical methods of Black Smoke and black carbon should give results with a simple 
relationship between them. Black carbon methods should ideally produce results similar to measures of EC, though there will always be differences due to the variation of mass extinction coefficient with soot particle size, due to the imperfect operational definition of $\mathrm{EC}$, and due to the optical effects of other particulate components present.

Accurate measurements of the composition of PM are vital to understanding the sources and evolution of PM in the atmosphere, and hence to implementing effective control measures to reduce its health effects. This is reflected in the requirement for compositional analysis (including EC and OC) in the recent EU Air Quality Directive (2008). Determining the health effects of specific components within PM also requires accurate measurements of those components. Aside from health effects, black carbon is also of interest because of its role as a short-lived but strong forcing agent for climate change (eg Shine at al., 2007). It is therefore important that such measurements as are available from national monitoring networks or elsewhere can be compared and interpreted with adequate knowledge of their strengths and deficiencies.

The results presented here relate primarily to the substantial volume of data available from the UK Particle and Black Smoke networks, funded by Defra (Department of the Environment, Food and Rural Affairs). The Particle network measured hourly organic carbon (OC) and elemental carbon (EC) using Rupprecht and Pattashnik (R \& P) 5400 automatic instruments at four sites between 2002 and 2007. Data from the period 2002 - 2004 was the subject of a paper by Jones and Harrison (Jones and Harrison, 2005). Since 2007, daily OC/EC measurements have been made by manual thermo-optical analysis of filter samples using a Sunset Laboratory Carbon Aerosol Analysis instrument. In parallel, long term daily measurements of Black Smoke have 
been made at many sites using the standard Black Smoke method. (ISO 9835, 1993). These instruments are currently being replaced by black carbon instruments (Magee AE22 aethalometers).

\section{$2 \quad$ Measurement methods}

\subsection{Manual OC/EC}

Daily samples were collected onto Pallflex Tissuequartz 47mm filters using a Partisol 2025 sampler fitted with a $\mathrm{PM}_{10}$ inlet and sampling at $1 \mathrm{~m}^{3} / \mathrm{hr}$. The filters are binderfree pure quartz filters that had been heat-treated by the manufacturer to remove trace organic compounds. This was checked by routine blank measurements. The filters from the rural Auchencorth Moss site were an exception, and the quartz filters used there had substantial OC blank content, though not EC, which was the parameter used for this set of results. After sampling, the filters were returned to NPL and kept in a fridge before analysis. $1.5 \mathrm{~cm}^{2}$ punches were then taken from the filter samples and analysed on a Sunset Laboratory Carbon Aerosol Analysis Lab Instrument. The protocol followed was a variation of the NIOSH protocol known as Quartz, which is described below. In an oxygen-free helium atmosphere, the sample is heated in four increasing temperature steps to remove organic carbon on the filter: $310^{\circ} \mathrm{C}$ for $70 \mathrm{~s}$, $475^{\circ} \mathrm{C}$ for $60 \mathrm{~s}, 615^{\circ} \mathrm{C}$ for $60 \mathrm{~s}$ and $870^{\circ} \mathrm{C}$ for $105 \mathrm{~s}$. As the organic compounds are volatilised, they are immediately oxidized to carbon dioxide in an oxidizer oven, which follows the sample oven. The flow of helium, containing the carbon dioxide, then goes to a methanator oven where the $\mathrm{CO}_{2}$ is reduced to methane. The methane is then detected by a flame ionisation detector (FID). Typically during this helium phase some organic compounds are converted to elemental carbon on the filter by pyrolysis. 
This pyrolitic conversion was continuously monitored by measuring the transmittance or reflectance of the sample using a laser.

After the sample oven is cooled to $525^{\circ} \mathrm{C}$, the pure helium eluent is switched to a $10 \%$ oxygen/helium mixture in the sample oven. The sample oven temperature was stepped up to $550^{\circ} \mathrm{C}$ for $60 \mathrm{~s}, 625^{\circ} \mathrm{C}$ for $60 \mathrm{~s}, 700^{\circ} \mathrm{C}$ for $60 \mathrm{~s}, 775^{\circ} \mathrm{C}$ for $60 \mathrm{~s}$, and $890^{\circ} \mathrm{C}$ for $110 \mathrm{~s}$. During this phase, both the original elemental carbon and that produced by the pyrolysis of organics during the first phase are oxidized to carbon dioxide. The TC value is based on the integrated FID response, while the split between OC and EC is calculated by assuming that the pyrolysed OC material has been removed when the transmittance or reflectance returns to its initial value. For technical reasons, in some cases both transmittance and reflectance data were available, while in others only transmittance or reflectance data were available.

After each sample run, a known volume and concentration of methane is injected into the sample oven. This provides a means of checking consistent operation of the instrument. The primary calibration of the entire Sunset instrument (for TC) is done by putting known amounts of sucrose on filters and analysing them using the full Quartz protocol. Machine blanks (every analysis session) and filter blanks (every six weeks) are also routinely measured to assess and correct for, if required, trace carbon contamination from the machine or from sampling. The Partisol samplers were calibrated annually for flow using traceable flowmeters, and the results of these calibrations used to produce the retrospectively ratified data sets.

It is well known that the determination of the $\mathrm{OC}$ and $\mathrm{EC}$ fractions can vary significantly depending on the choices of heating protocol and optical correction, and also that the presence of mineral oxides, for example, can affect the oxidation of carbonaceous material and hence affect the results (Chow et al, 2001; Chow et al, 
2005). These are active areas of study, for example within the EUSAAR project mentioned above, that do not directly affect the conclusions formed here, which relate to the comparability of existing data sets within UK Networks rather than the much broader topic of OC and EC determination.

\subsection{Automatic OC/EC}

The Rupprecht and Pattashnick (latterly Thermo) 5400 Ambient Carbon Particulate Monitor collected hourly samples through a $\mathrm{PM}_{10}$ inlet operating at $1 \mathrm{~m}^{3} / \mathrm{hr}$. The particulate matter is collected on an internal impactor with a 50\% cut off point around $100 \mathrm{~nm}$, so that, unlike simple filter samples, only particles larger than around $100 \mathrm{~nm}$ in aerodynamic diameter are collected and measured. After sampling, the impactor is heated to two temperatures $\left(\sim 350^{\circ} \mathrm{C}\right.$ and $\left.\sim 750^{\circ} \mathrm{C}\right)$ in ambient air to obtain measures of organic and total carbon content through the amount of carbon dioxide generated. The analysis is performed using an infrared LiCor detection system to measure the amount of $\mathrm{CO}_{2}$. Elemental carbon is calculated by subtraction of the organic carbon content from the total carbon content. There is no optical correction for the pyrolysis of organics during the first heating phase. For this comparison, daily mean concentrations were calculated from the hourly measured values.

Several differences are to be expected between data obtained by this automatic method compared to those from the manual method described in the previous section. The impactor collection means that the many particles smaller than $100 \mathrm{~nm}$ in size will not contribute to the result. As fresh diesel particles are typically $30 \mathrm{~nm}$ in size this is likely to be very significant at kerbside locations. The less sophisticated split between OC and EC will inevitably lead to different results from the thermo-optical method. More fundamentally, although the LiCor sensor can be calibrated with a known $\mathrm{CO}_{2}$ mixture, the whole system (including the impaction and heating stages) is 
not amenable to direct calibration with a known amount of carbon material. Therefore, unlike the manual method, even the accuracy of the TC measurements is difficult to assess. (The manual method TC calibration does not include sampling losses through the inlet, filter collection efficiency or sampling artefacts such as losses of semivolatile organic compounds or collection of volatile organic compounds. Filter collection efficiencies are high for all particle sizes, so that collection is much less of a problem for the manual method than for the impactor. Losses in pipework are a separable issue affecting both methods. Sampling artefacts are largely a matter of convention until a reference method is defined.)

Studies performed by the US-EPA have shown that results from the 5400 instrument did not agree well with results obtained using the NIOSH 5040 protocol (US-EPA, 2005). They found significant negative bias in the R\&P 5400 TC, OC, and EC data at all of their five sites, with regression slopes typically 0.4 to 0.6 , combined with poor reproducibility (termed predictability in the document). Results from this instrument should therefore be treated a priori with more caution than those from the other methods.

\subsection{Black Smoke}

The Black Smoke method involved pumping air at a rate of $0.084 \mathrm{~m}^{3} / \mathrm{hr}$ through a known area of Whatman Number 1 cellulose filter paper, and measuring the darkness of the stain by a measurement at the National Physical Laboratory of its reflectance of white light - the reflectance being relative to the light reflected by a clean filter of the same material. The inlet, essentially an upturned funnel, provides crude size selection, removing particles above around $4.5 \mu \mathrm{m}$ (McFarland et al, 1982). The reflectance measurements were made using an EEL M43D Smokestain Reflectometer. The 
reflectance, volume and area are converted to a Black Smoke Index using a standard table based on ISO 9835 (1993). There are clear theoretical grounds (Quincey, 2007), for considering that the Black Smoke Index $I_{B S}$ is related to estimates of black carbon $C_{B C}$, as measured by an aethalometer (below), following the relation:

$$
C_{B C}\left[\mu g \cdot m^{-3}\right]=\sqrt{4.18 I_{B S}+59.6}-7.72
$$

In the presentations of data below, Black Smoke results, which would otherwise appear anomalously high compared with other measures of elemental or black carbon, have been converted to "black carbon" results using this equation.

The sampler flow was calibrated annually using a traceable flowmeter. The reflectometer protocol involves zeroing the instrument for each measurement, while the reflectance scale was checked against tiles certified at NPL.

\subsection{Aethalometer}

Aethalometers quantify "black carbon" on filter samples based on the transmission of light through a sample. The sample is collected onto a quartz tape, and the absorption coefficient of the sample is measured by a single pass transmission of light through the sample, measured relative to a clean piece of filter. $880 \mathrm{~nm}$ wavelength light was used. The absorption coefficient $\alpha\left[\mathrm{m}^{-1}\right]$ is calculated from the transmission, area and volume of the sample, and converted to a black carbon concentration $\mathrm{C}_{\mathrm{BC}}$, as a first approximation, using the mass extinction coefficient $\alpha_{\text {atn }}$.

With the Magee AE22 Aethalometer instrument used for the data below, the value used is $\alpha_{\text {atn }}=16.6 \mathrm{~m}^{2} / \mathrm{g}$ (taken from Magee literature). Instrument flow was calibrated with traceable flowmeters. The aethalometer operated at Marylebone Road had a 
$\mathrm{PM}_{2.5}$ inlet, while the one at Auchencorth Moss has an aerodynamic size cutoff at the inlet of $3.3 \mu \mathrm{m}$.

\section{Parallel measurements of Black Smoke, aethalometer black carbon, automatic EC/OC and manual EC/OC at a London kerbside site, September to December 2006}

The results presented here are selected from a larger study reported as the Marylebone Road Aethalometer Trial Report (Green et al, 2007). The aim of the larger study was primarily to evaluate aethalometer instruments for measurements of Black Smoke. Some results comparing an aethalometer with an automated Black Smoke instrument (ETL SX200) were published within the paper by Quincey (2007). In this paper the aethalometer results are compared with those from the other instruments used within measurement networks.

Marylebone Road is a kerbside monitoring site in central London (UK ordnance survey grid reference 528120, 182000). Marylebone Road is a major route in and out of Central London, running north-east to south-west and carrying approximately 90,000 vehicles per day. The tall buildings on either side form a broad street canyon about $40 \mathrm{~m}$ across. The monitoring cabin is located $1 \mathrm{~m}$ from the kerb on the southern side of the road.

Due to space limitations it was not possible to site all the equipment exactly equidistant from the kerb. Although disparities were kept to a minimum, the fact that traffic emissions dominate measurements at this site means that small differences in distance from the road need to be considered. The exact distances from the kerb were: 8-port sampler (Black Smoke), 1.50m; R\&P Automatic Carbon Monitor 5400, 2.23m; 
Magee Aethalometer AE-21, 2.97m; Partisol 2025 (used to collect samples for analysis by Sunset) $2.97 \mathrm{~m}$.

Parallel results are available for a period of about three months between September and December 2006. They are presented here separately as "elemental” carbon results, and "organic" carbon results. The "elemental" results contain the automatic and manual EC, the aethalometer black carbon, and the Black Smoke, and these are shown in Figure 1. The Black Smoke results have been converted from Black Smoke Index to black carbon $\left(\mu \mathrm{g} / \mathrm{m}^{3}\right)$ as described above. The pyrolysis corrections on the manual OC/EC results were calculated using the transmittance data.

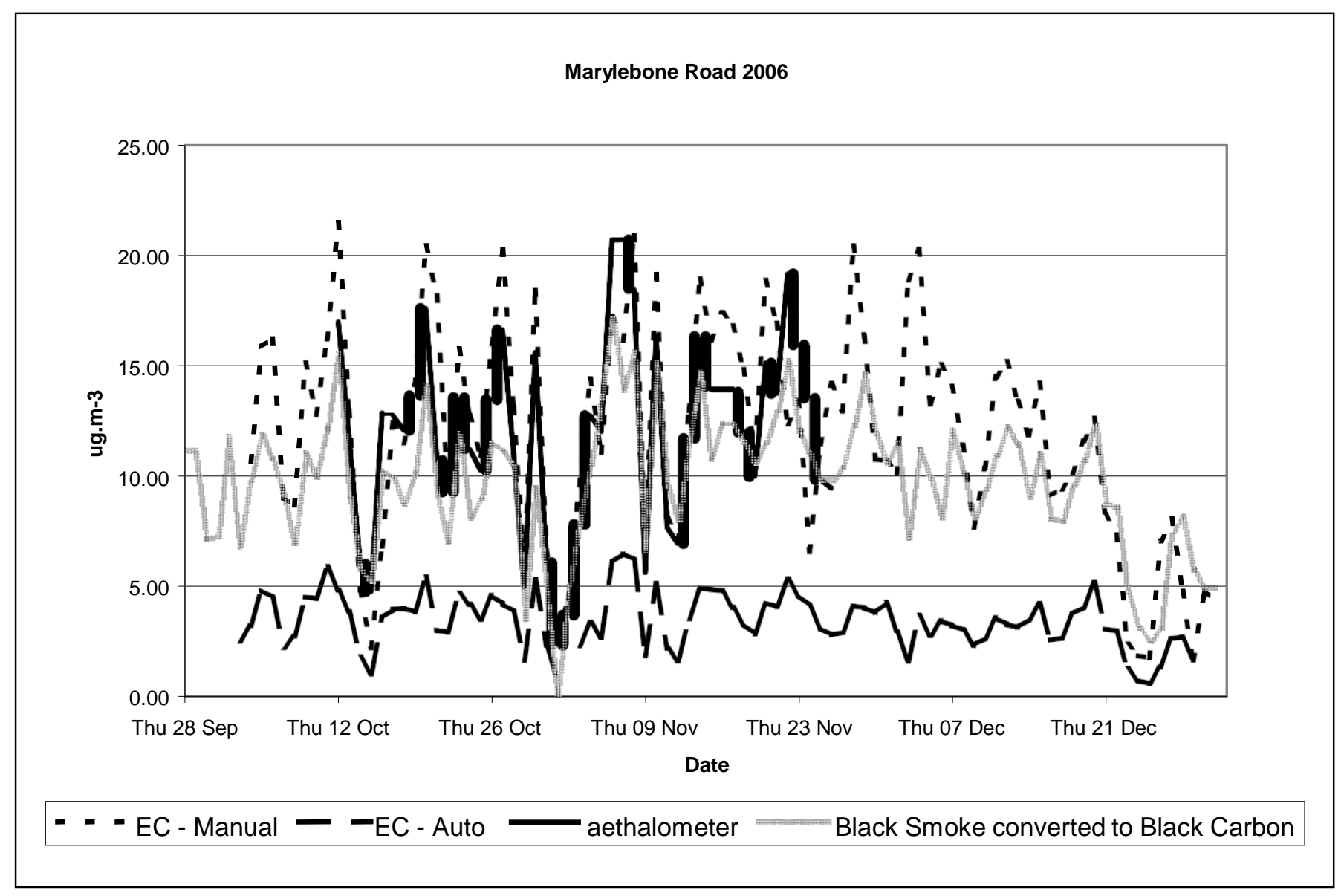

Figure 1: Daily mean "elemental" carbon concentrations from a London kerbside site. 
There is reasonable quantitative agreement between the manual EC, the aethalometer, and the converted Black Smoke, especially in view of the fact that one instrument is based on chemical analysis and the other two are based on optical properties, and the inhomogeneities associated with a kerbside site. The major discrepancy is between these instruments and the automatic EC, which typically underreads by approximately a factor 3. The likely explanation for this is that at the kerbside, a large proportion of the elemental carbon is in the form of particulates with an aerodynamic diameter less than $100 \mathrm{~nm}$, which would not be collected by the sampling impactor of the R \& P automatic instrument, whereas the three other methods collect smaller particles efficiently on fibrous filters.

Figure 2 shows the "organic" carbon results. In contrast to the elemental results, there is much better agreement between the manual and automatic methods. However, a step change can be observed in the relationship between the two sets of results, apparently coinciding with a six-monthly service visit on $2^{\text {nd }}$ November. The results either side of the service have been scaled using the routine calibrations for $\mathrm{CO}_{2}$ at the site, but this has not removed the effect. We interpret this as an indication that the lack of direct full-instrument calibration in the automatic instrument makes reliable QA/QC difficult. 


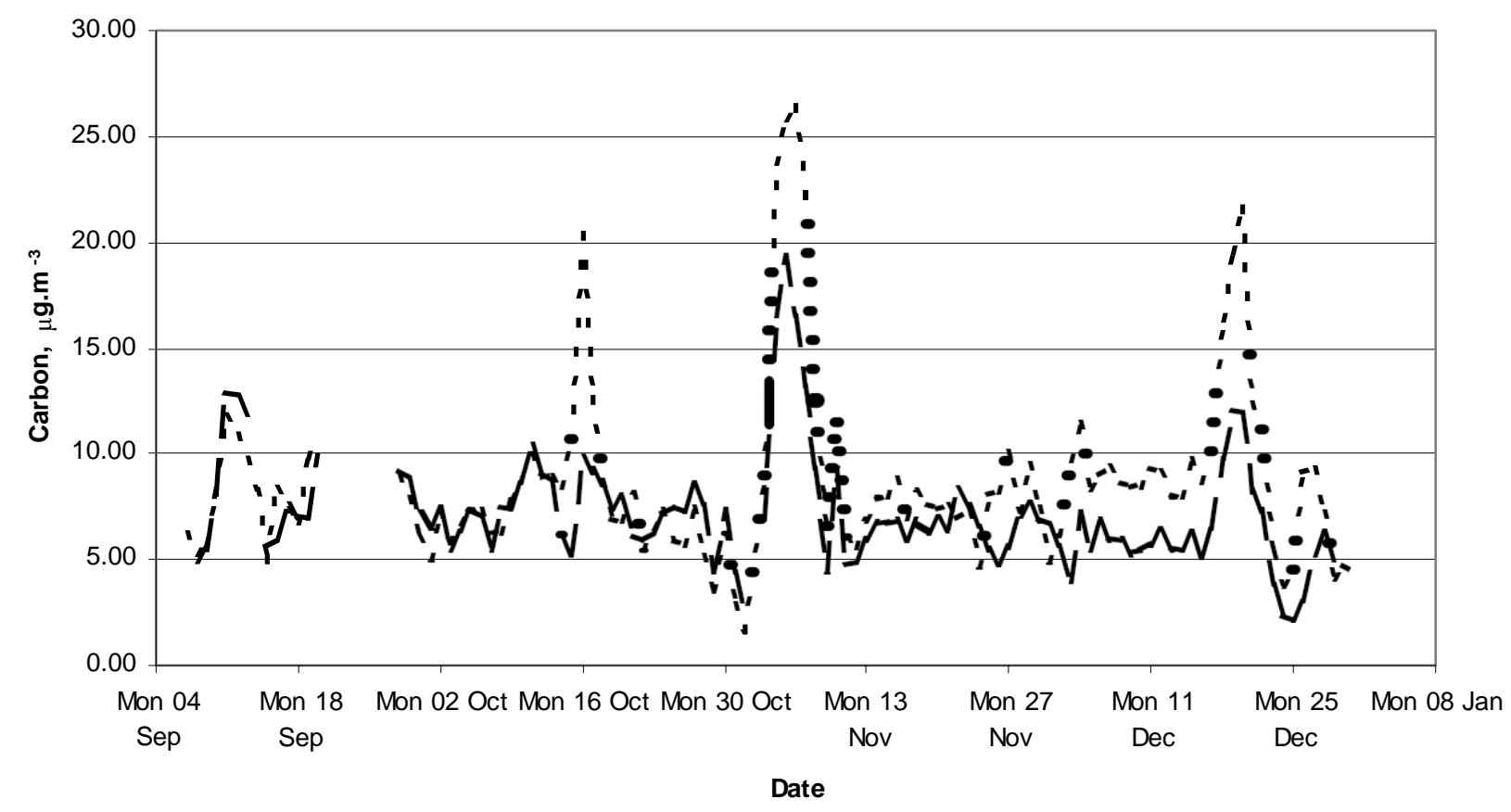

- - - Manual OC — - Auto OC

Figure 2: Daily mean organic carbon concentrations from a London kerbside site.

The imperfect methods for distinguishing between "organic" and "elemental" carbon in these instruments means that one would expect the total carbon (TC) to agree more closely than the separate fractions. In this case, the large difference in the EC results, of around $10 \mu \mathrm{g} / \mathrm{m}^{3}$, means that the total carbon results from the two instruments differ by about a factor of 2 , with around $20 \mu \mathrm{g} / \mathrm{m}^{3}$ TC from the manual method and $10 \mu \mathrm{g} / \mathrm{m}^{3}$ from the automatic.

\section{Parallel measurements of automatic EC/OC and manual EC/OC (August} to October 2006), and Black Smoke and automatic EC (March to April 2007) at a London background site.

The North Kensington site is classified as an urban background site, in the grounds of a school in a residential area $7 \mathrm{~km}$ to the west of central London. Further details about the monitoring site can be found through the website www.bv-aurnsiteinfo.co.uk. 
The site is expected to be much less dominated by traffic emissions than Marylebone Road, with lower particulate concentrations and with larger elemental carbon particles due to agglomeration.

The parallel data is in two parts: firstly, measurements from manual and automatic OC/EC instruments over the period August to October 2006, and secondly from automatic OC/EC and Black Smoke in March and April 2007. Only the EC results from this latter period are presented.

Results from the manual and automatic EC and OC are shown in Figures 3 and 4. The pyrolysis corrections on the manual OC/EC results were calculated using the transmittance data, as before.

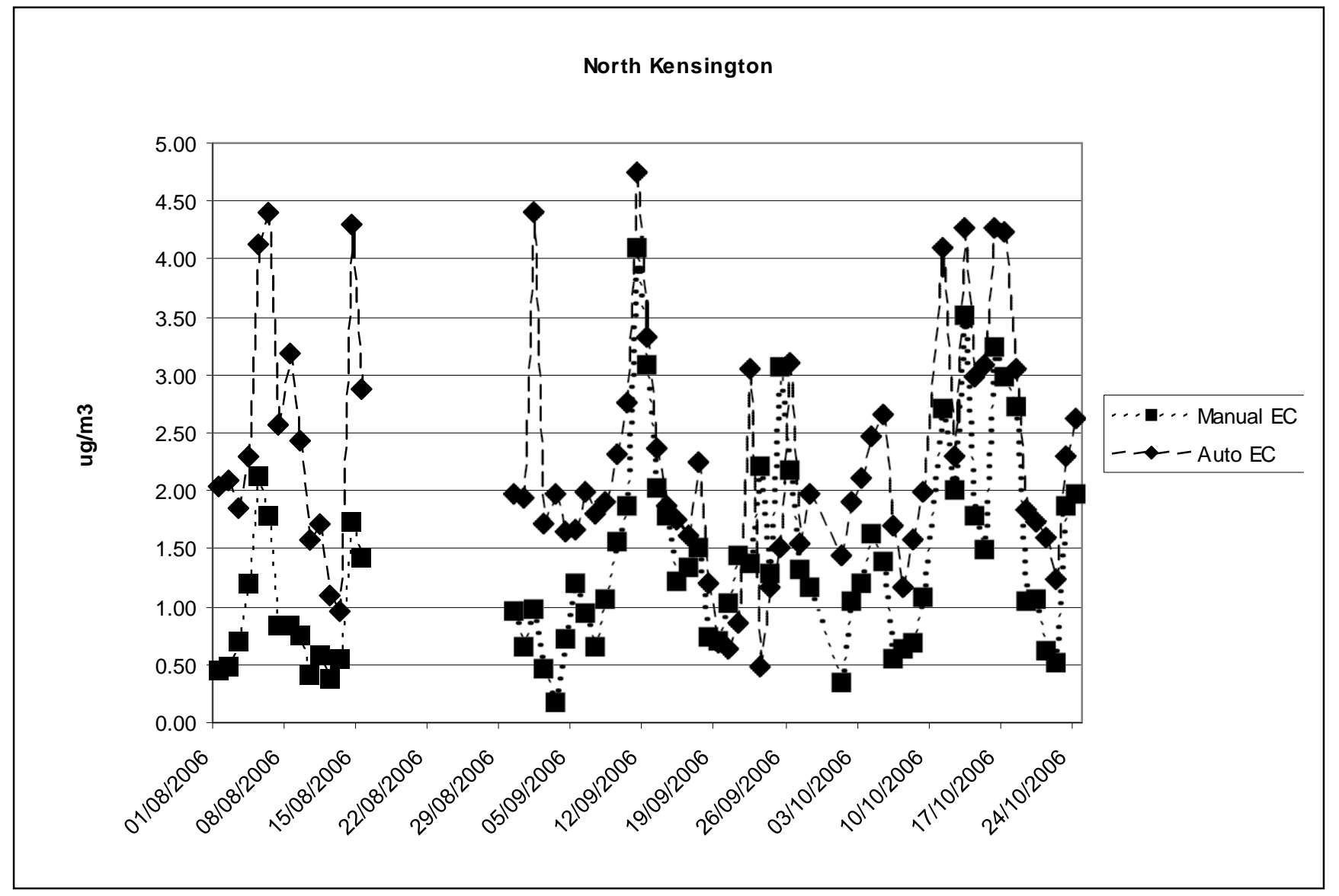

Figure 3: Daily mean elemental carbon concentrations at a London background site. 
Two differences from the Marylebone Road data are immediately apparent from the results - concentrations are very much lower (around $1.5 \mu \mathrm{g} / \mathrm{m}^{3}$ for manual EC, compared with around $12 \mu \mathrm{g} / \mathrm{m}^{3}$ at Marylebone Road, with around $4 \mu \mathrm{g} / \mathrm{m}^{3}$ and 8 $\mu \mathrm{g} / \mathrm{m}^{3}$ respectively for manual $\mathrm{OC}$ ), while the automatic results do not differ from the manual ones in the same way. The automatic EC results do not under-read the manual ones by a factor of 3, but rather tend to over-read by an average of around $40 \%$, although the difference is quite variable, and the period $12^{\text {th }}$ to $20^{\text {th }}$ September shows reasonable agreement. Automatic OC is about a factor of 2 larger than the manual OC, unlike the near parity at Marylebone Road. Total carbon with the automatic method at around $10 \mu \mathrm{g} / \mathrm{m}^{3}$ is typically a factor of 2 larger than the manual one (5 $\left.\mu \mathrm{g} / \mathrm{m}^{3}\right)$, in direct contrast to the results from Marylebone Road.

\section{North Kensington}

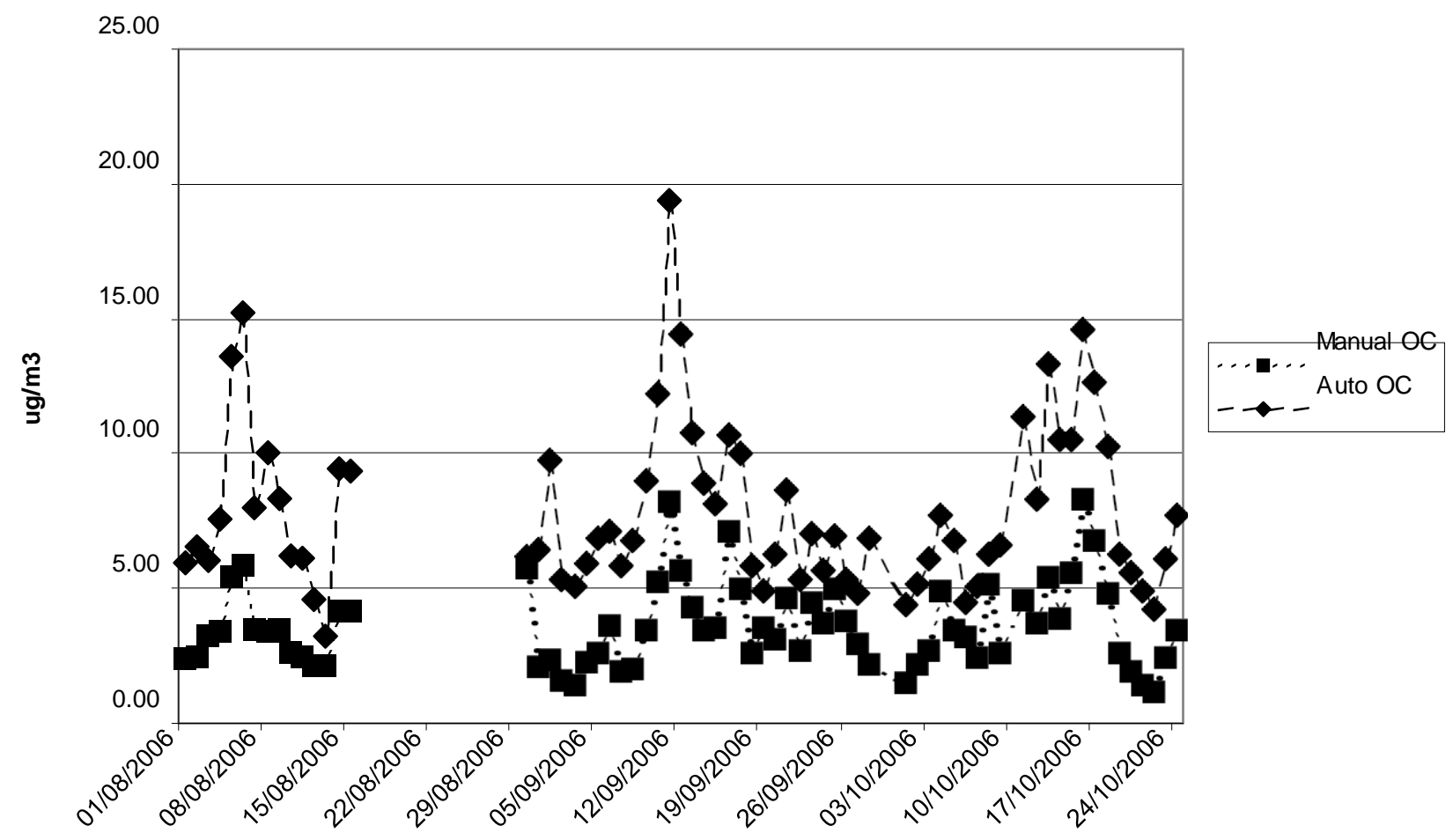

Figure 4: Đaily mean organie earbon eoneentrations at a London baekground site. 
These differences have no obvious explanation. Selected repeat measurements made on the manual OC/EC instrument using second samples cut from the same filters showed good agreement, and the more direct QA/QC for the manual method means that there is little reason to doubt their basic accuracy, especially for TC. The much higher TC results from the automatic method are therefore hard to account for, especially as the automatic instrument will be sampling a narrower particle size range. We interpret this as an example of the generic inability to characterise the performance of the automatic instrument in a satisfactory way. Other anomalous results from these instruments have been noted (eg Hayman et al, 2008 and US-EPA, 2005). It is clear that while these instruments can produce useful data within their inherent limitations, the inability to check whether the instrument is functioning normally is a major barrier to confident application of their data.

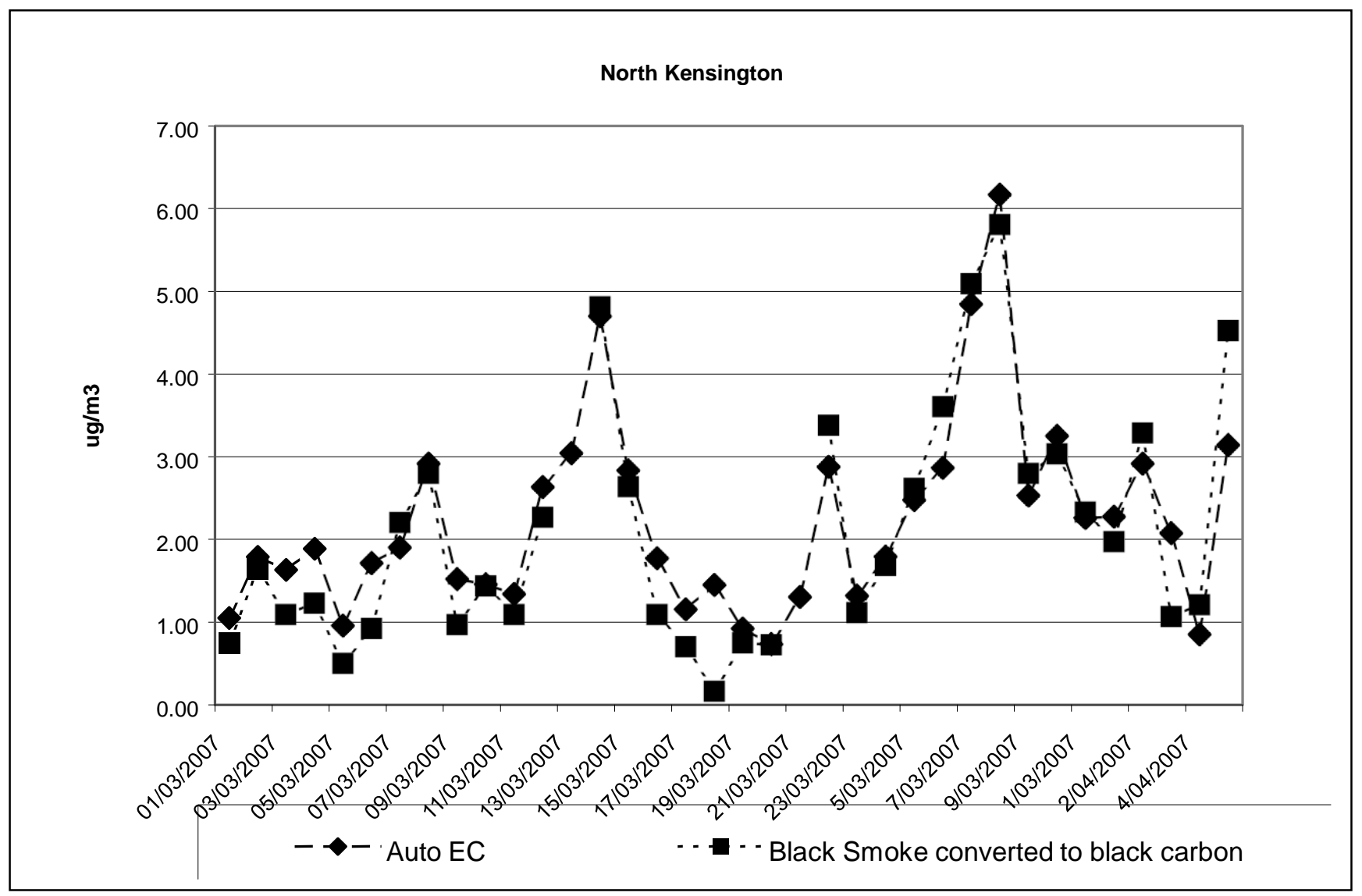

Figure 5: Daily mean elemental carbon concentrations and converted Black Smoke measurements at a London background site. 
Figure 5 shows the second set of data from the North Kensington site, a period of several weeks soon after the start of Black Smoke measurements at the site and shortly before the end of automatic OC/EC measurements, starting about six months after the previous data set.

The agreement is remarkably close. One can speculate that the automatic OC/EC was operating, like the period from $12^{\text {th }}$ to $20^{\text {th }}$ September, to give results close to parity with manual EC results, and hence close to the black carbon results. However, in view of the variability of performance of the automatic instrument noted previously, there is likely to be an element of luck in the agreement, and it would be difficult to extrapolate from this observation to other sets of results.

5 Parallel measurements of aethalometer black carbon and manual EC at a rural Scottish site, June to December 2006.

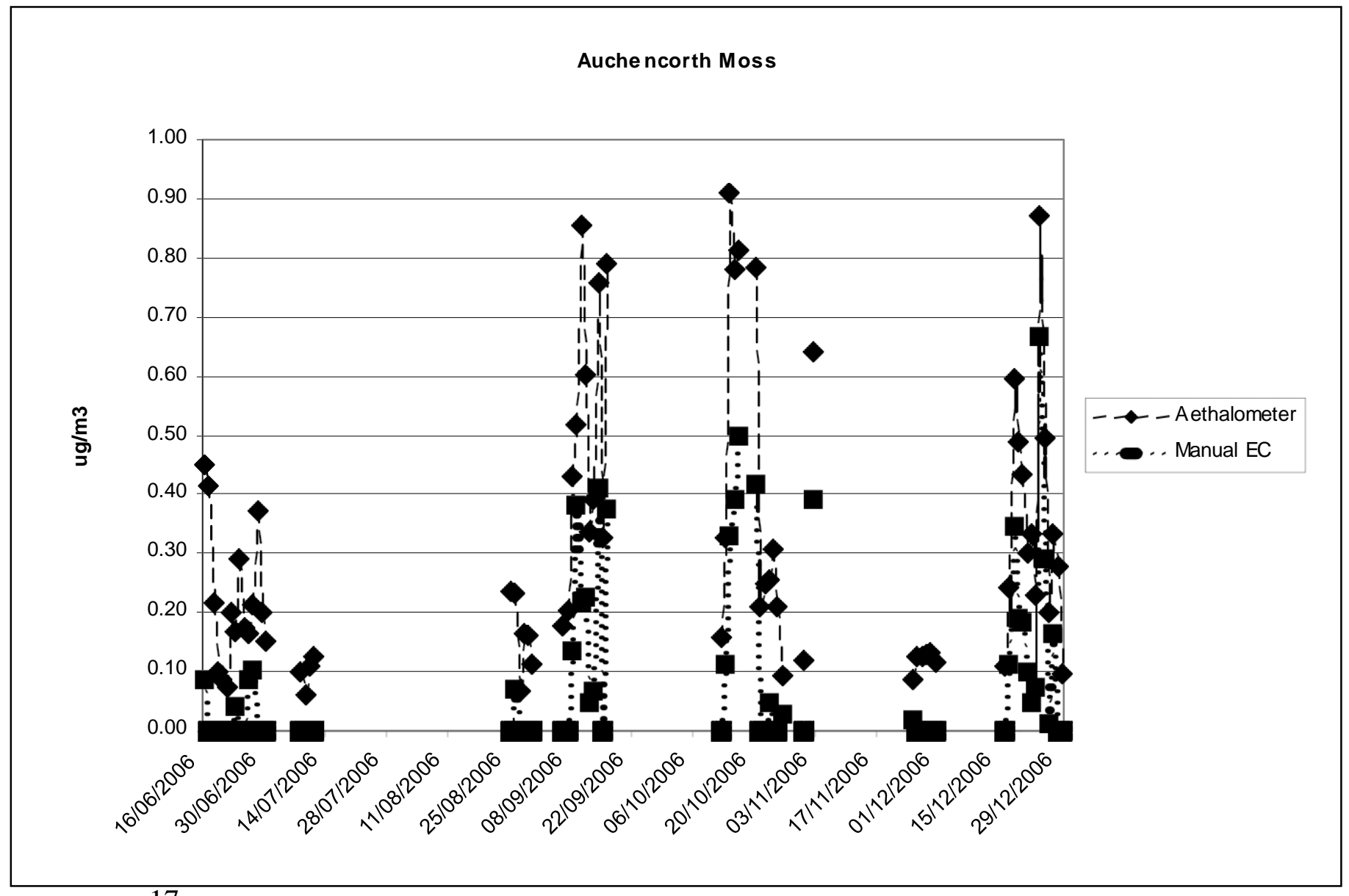


Figure 6: Daily mean aethalometer and elemental carbon concentration measurements at a rural Scottish site.

The final data set is from the EMEP rural background site at Auchencorth Moss in Scotland. The site is situated $3 \mathrm{~km}$ from the nearest road in the middle of a peat bog, 8 km south-west of the town of Penicuik, SE Scotland (lat 55:44:49N, long 3:39:22W). More details can be found at http://www.heavymetals.ceh.ac.uk/sites/site_auc.htm.

There are two significant differences in the measurement method for manual EC/OC from the previous data. Firstly, the quartz filters were not pre-baked. Blank measurements showed negligible amounts of EC on the filters but variable amounts of OC comparable in quantity with the sampled OC. Secondly, for these measurements the pyrolysis correction was made with the reflectance data, as the transmittance data was not available. It is widely found that EC data using a reflectance correction will be higher than those found using a transmittance correction (eg Chow et al, 2001), as the correction for pyrolysed material tends to be smaller.

The concentrations are very low, less than $1 \mu \mathrm{g} / \mathrm{m}^{3}$, and it is notable that in this case the manual method significantly underreads the aethalometer data, to the extent that many of the manual EC data are zero. Simple regression analysis gives a relationship between the two data sets of $\mathrm{EC}($ aeth $)=1.33 \mathrm{EC}($ manual $)+0.2 \mu \mathrm{g} / \mathrm{m}^{3}$, with $\mathrm{R}^{2}=$ 0.79 .

As the darkening of the filter registered by the aethalometer is likely to be real, this suggests that the manual EC method is overcorrecting for pyrolysis, even when using the reflectance data. While the TC measurement from the manual method should be correct, this would imply that the conventional split between EC and OC using common thermo-optical methods under-reads the "true" elemental carbon (as indicated by the presence of soot), whether a transmittance or a reflectance charring 
correction is used. These effects would probably have little consequence for roadside measurements, but could be very significant for rural sites.

\section{Conclusions}

The purpose of this paper is to highlight some measurement issues associated with ambient particulate organic, elemental and black carbon, as monitored at UK network sites, to provide background information to aid interpretation of the data. The examples presented are not comprehensive, but are relevant to this active field of study both in the UK and more widely.

The main conclusions are that, from the results available, the R \& P 5400 instruments greatly under-read EC and TC at kerbside sites, probably due to the fact that the smaller particles are not sampled by the instrument; the R \& P 5400 instrument is inherently difficult to characterise, so that all quantitative results need to be treated with caution; both aethalometer and Black Smoke (converted to black carbon) measurements can show reasonable agreement with elemental carbon results; and manual thermo-optical OC/EC results using the "Quartz" protocol may under-read EC (and hence overread OC), whether either transmittance or reflectance is used for the pyrolysis correction, and this effect is significant at rural sites.

\section{Acknowledgements}

The authors would like to thank the UK's Department for Environment, Food and Rural Affairs (Defra) for funding the Marylebone Road trial and the EMEP monitoring site at Auchencorth Moss; and the UK's Department for Innovation, Universities and Skills (DIUS) for support through its Chemical and Biological Metrology Programme. 


\section{References}

AQEG, 2005. Particulate Matter in the United Kingdom, Air Quality Expert Group, UK Department for Environment, Food and Rural Affairs.

Chow, J.C., Watson, J.G., Crow, D., Lowenthal, D.H., Merrifield, T., 2001.

Comparison of IMPROVE and NIOSH carbon measurements, Aerosol Science and Technology 34 23-34.

Chow, J.C., Watson, J.G., Chen, L-W.A., Paredes-Miranda, G., Chang, M-C.O., Trimble, D., Fung, K., Zhang, H., Yu, J.Z., 2005. Refining temperature measures in thermal/optical carbon analysis; Atmos. Chem. Phys. Discuss., 5, 4477-4505.

Green, D., Alexander, J., Fuller, G., Quincey, P., Butterfield, D., 2007. Marylebone Road aethalometer trial report, available through the Defra website www.airquality.co.uk.

Hansen, A.D.A., Rosen, H., and Novakov, T., 1984. The aethalometer - an instrument for the real-time measurement of optical absorption by aerosol particles, Science of the Total Environment 36, 191.

Hayman, G., Yardley, R., Quincey, P., Butterfield D., Green, D., Alexander, J., Johnson, P., Tremper, A., 2008. CPEA 28: Airborne Particulate Concentrations and 
Numbers in the United Kingdom Annual Report 2007, available through the Defra website www.airquality.co.uk.

EU Directive 2008/50/EC on ambient air quality and cleaner air for Europe, available through http://eur-lex.europa.eu/en/index.htm.

Jones, A.M. and Harrison, R.M., 2005. Interpretation of particulate elemental and organic carbon concentrations at rural, urban and kerbside sites, Atmospheric Environment 39 7114-7126.

Quincey, P., 2007. A relationship between Black Smoke Index and Black Carbon concentration, Atmospheric Environment 41 7964-7968.

ISO 9835, 1993. Methods for measurement of air pollution - part 11: determination of a Black Smoke index in ambient air.

McFarland, A.R., Ortiz, C.A., Rodes, C.E., 1982. Wind tunnel evaluation of the British Smoke Shade sampler, Atmospheric Environment 16 325-328.

Shine, K.P., Berntsen, T.K., Fuglestvedt, J.S., Skeie, R.B., Stuber, N., 2007. Comparing the climate effect of emissions of short- and long-lived climate agents, Philosophical Transactions of the Royal Society A 365 1903-1914. 
United States Environmental Protection Agency, 2005. Comparison of integrated filter and semi-continuous measurements of PM2.5 nitrate, sulfate and carbon aerosols in the Speciation Trends Network (STN), EPA 454/R-05-004.

Turpin, B.J., Liu, S-P., Podolske, K.S., Gomes, M.S.P., Elsenrelch, S.J., McMurry, P.H., 1993. Design and Evaluation of a Novel Diffusion Separator for Measuring Gas/Particle Distributions of Semivolatile Organic Compounds, Environ. Sci. Technol. 27, 2441-2449

Eatough, D.J., Wadsworth, A., Eatough, D.A., Crawford, J.W., Hansen L.D., Lewis, E.A., 1993. A multiple-system, multi-channel diffusion denuder sampler for the determination of fine-particulate organic material in the atmosphere, Atmospheric Environment. Part A, General topics 27 1213-1219

(C) Crown copyright 2009. Reproduced by permission of the Controller of HMSO 